\title{
Perceptions of community-based human milk banks before and after training in a resource-limited South African setting
}

\author{
H E Goodfellow, ${ }^{1} \mathrm{MB}$ ChB; P Reimers, ${ }^{2}$ MTech (Nursing); K Israel-Ballard, ${ }^{3} \mathrm{DrPH}$; A Coutsoudis, ${ }^{2} \mathrm{PhD}$ \\ ${ }^{1}$ Department of Paediatrics and Child Health, School of Clinical Medicine, University of KwaZulu-Natal, Durban, South Africa \\ ${ }^{2}$ Department of Paediatrics and Child Health, Nelson Mandela School of Medicine, University of KwaZulu-Natal, Durban, South Africa \\ ${ }^{3}$ Maternal and Child Health and Nutrition Global Program, Seattle, USA
}

Corresponding author: H E Goodfellow (heloise_vdb@hotmail.com)

\begin{abstract}
Background. Human breastmilk provides gold standard nutrition and immunological support to infants. For low birth weight, HIVinfected, HIV-exposed or otherwise vulnerable babies, it can mean the difference between life and death. When a mother's own milk is not available, safe, donated human breastmilk is an excellent alternative. High rates of under-5 mortality have prompted the South African (SA) Ministry of Health to commit to scaling up human milk banks in key health facilities. Community-based human milk banks (CBHMBs) have the potential to complement these efforts, but there is little research on the feasibility and acceptability of this approach.

Objective. To determine mothers' perceptions of breastfeeding and CBHMBs, and to ascertain how training could affect those perceptions. Methods. A total of 40 black mothers in KwaZulu-Natal, SA, participated in a survey on breastfeeding and human milk banks (HMBs) prior to commencing a breastfeeding peer-counselling training course that included information on HMBs. The survey was re-administered following the completion of the module on HMBs. The questionnaire was repeated $\sim 4$ months later.

Results. Following training, significant changes were observed in mothers' knowledge and perceptions around donor milk and safety. No significant changes were observed in reports of what these mothers presumed the community's perceptions around donor milk banking were. Conclusion. Education of mothers can play an important role in supporting CBHMBs by improving mothers' perceptions and acceptance of breastfeeding, donor milk, and milk banking. Changing community concerns around HMBs will require more than just changing the perceptions of mothers.
\end{abstract}

S Afr J Child Health 2016;10(1):83-86. DOI:10.7196/SAJCH.2016.v10i1.1103

Human breastmilk is the gold standard in infant nutrition. It is particularly crucial for vulnerable, orphaned, or low birth weight infants and those exposed to, or infected with HIV. It provides powerful nutritional and immunological protection and reduces mortality due to infectious diseases. ${ }^{[-3]}$ It also lowers the risk of necrotising enterocolitis among preterm, low birth weight infants. ${ }^{[4,5]}$ Globally, the promotion of exclusive breastfeeding is seen as an important public health priority ${ }^{[6]}$ When a mother's own breastmilk is not available, the World Health Organization recommends donated human breastmilk (DHM) as the best alternative. ${ }^{[7]}$

As a result, countries worldwide are establishing human milk banks (HMBs) to collect, pasteurise, and provide safe DHM to vulnerable infants. In 2011, the South African (SA) Ministry of Health committed to promoting and supporting HMBs in postnatal wards and neonatal intensive care units (NICUs) to reduce neonatal and postnatal morbidity and mortality. ${ }^{[8]}$ The use of DHM in NICUs was seen as a temporary measure while the mothers are supported to establish their own breastmilk supply, and therefore HMBs were seen as part of the strategy to support and promote breastfeeding.

SA's AIDS epidemic continues to exact a devastating toll on mothers, leaving many infants and children orphaned. According to a 2011 census, $18.8 \%$ of SA children between birth and 17 years of age had lost one or both parents to the disease. In KwaZulu-Natal (KZN), one of the poorest provinces in the country, this number rose to more than a quarter $(27 \%) .{ }^{[9]}$ Given the poor socioeconomic conditions in many parts of $\mathrm{KZN}$, the provision of adequate nutrition to infants younger than 6 months of age is a major dilemma. This has stimulated the concept of community-based HMBs (CBHMBs), which operate outside a health facility, in locations such as homes for orphans or other community centres. These CBHMBs have the potential to reduce infant mortality by expanding access to lifesaving human milk to vulnerable infants beyond those only in a neonatal ward. Although research has demonstrated that HMBs in NICUs are acceptable and feasible in $\mathrm{SA}^{[10]}$ and beyond, ${ }^{[5,11-14]}$ there is, to our knowledge, no data recording the acceptability or experiences of CBHMBs.

Successfully increasing CBHMBs would require an understanding of the benefits of breastfeeding and knowledge and acceptance of donor milk banking. Rigorous quality-control systems would also need to be established to ensure the safety of donor milk. The objective of this study was to determine mothers' perceptions of CBHMBs and how education and training could affect or improve their opinions.

\section{Methods}

The research team interviewed women who were participating in a community nutrition programme being conducted at the Cato Manor Clinic in an informal settlement in Durban in KZN. This province has the third-highest unemployment rate $(33 \%)$ in SA. ${ }^{[9]}$

The community nutrition programme conducted at the clinic included an interactive breastfeeding peer counselling training course, which was offered to mothers who had recently delivered babies. The course not only assisted them with their own breastfeeding practices but also looked to prepare them to become breastfeeding counsellors in the community. The mothers were enrolled in the course when their infants were approximately 6 weeks of age. One-hour long sessions were held every 2 weeks until the infants reached 5 months of age. Thereafter the sessions were held monthly until the infants were 12 months of age.

The research team verbally administered questionnaires to mothers when they first enrolled in the programme and before they received 
any training sessions. The same questionnaires were re-administered to these mothers $\sim 22$ weeks post delivery. At this stage they had completed the HMB training module and had been part of the training course for about 4 months. The questionnaires, which were administered by a research assistant fluent in English and isiZulu, contained questions on demographics, as well as questions which aimed to ascertain the acceptability and safety of donor breastmilk and the willingness of the mothers to donate breastmilk and to set up a CBHMB. Questions also attempted to elicit how mothers thought the community would react to or accept the concept of mothers donating breastmilk and the fact that infants, including orphans, would be receiving DHM. Most of the questions were not open ended and the questionnaire was developed from scratch since there was no appropriate validated questionnaire available for use. The questionnaire was piloted with five mothers of similar background before it was finalised.

A convenience sample of 40 women was approached for permission to be enrolled into an evaluation of the effect of the training programme on perceptions around human milk banking. These were the last mothers to be enrolled into the peer counsellor training programme (March - May 2013). Only 38 of the women who had completed the training programme were available to complete the post-training questionnaire. Although Cato Manor Clinic serves a predominantly isiZulu-speaking community, $\sim 80 \%$ of the women are able to speak English. The training programme was limited to women who could read and speak simple English since many of the training materials were in English. The discussion sessions during the training sessions were, however, conducted in isiZulu.

All mothers signed consent forms before participating in the study and the data were held confidentially. The study was approved by the Biomedical Research Ethics Committee of the University of KwaZulu-Natal (Ref: BE 210/12).

\section{Results}

\section{Socioeconomic data}

Data from the questionnaire show that most survey respondents were single, unemployed, had little education, and were living in homes with poor sanitation (Table 1).

\section{Mothers' perceptions}

Survey questions about DHM focused on donor milk safety and personal and community views of donor milk use. Some significant changes in knowledge and perceptions were observed following the training. Initially, most mothers were hesitant to become involved in donating, feeding, or banking breastmilk. The data showed that post training, mothers' perceptions had changed on most, but not all, topics (Fig. 1).

\section{Willingness to donate breastmilk}

Data collected before training showed that a narrow majority of mothers, 23 of 40 (58\%), were willing to donate breastmilk. After training, this improved significantly $(p<0.05)$ with most mothers, 33 of $38(86.8 \%)$ being willing to be donors.

\section{Willingness to feed own baby donated breastmilk}

Initially, mothers largely rejected the possibility of feeding their own baby with milk donated by another mother, with only 12 of the $40(30 \%)$ indicating willingness to do so. Following training, however, this increased significantly $(p<0.05)$, with 28 of the 38 $(73.7 \%)$ mothers prepared to feed their own baby donated breastmilk.

\section{Acceptability of CBHMBs to the mothers themselves}

Before training, some mothers, 23 of 40 (57.5\%), considered the idea of them setting up CBHMBs acceptable. Following training this did not change significantly ( $p=0.21)$, with 27 of the 38 (71.1\%) finding the concept acceptable.

\section{Community perceptions}

This question revealed that mothers continued to hold the view that although their perceptions may have changed, the wider community perceptions around DHM remained largely unfavourable. In the initial questionnaire 24 of the $40(60 \%)$ mothers felt the community would view feeding a baby donor milk as unacceptable. In the posteducation questionnaire the findings showed a non-significant difference ( $p=0.65$ ), with 21 of the $38(55.3 \%)$ mothers reporting this. Similarly before training, 20 of the $40(50 \%)$ mothers indicated that the community would perceive breastmilk donors as 'not acceptable' and after training, 21 of the $38(55.3 \%)$ held this view ( $p=0.73)$.

\section{Discussion}

To the best of our knowledge this is the first survey measuring perceptions of mothers to CBHMBs, before and after education around HMBs. The findings of pre-training perceptions are similar to early findings from Nigeria, where the major concern expressed by mothers with regard to the feeding of donor breastmilk to their own baby was that it would not be safe. ${ }^{[13]}$ Studies on the

Table 1. Sociodemographic characteristics of respondents $(N=40)$

\begin{tabular}{ll}
\hline Characteristic & $\boldsymbol{n}(\%)$ \\
\hline Age (years), mean (SD) & $25.4(5.6)$ \\
$15-24$ & $23(57.5)$ \\
$25-34$ & $14(35.0)$ \\
$\geq 35$ & $3(7.5)$
\end{tabular}

Marital status

Single

Married

Living with partner

Education

Primary education incomplete

Completed primary education

$17(42.5)$

Completed secondary education

Some tertiary education

Employment

Unemployed

Employed

On maternity leave

Dependent on social welfare grant

Yes

No

Water

Tap in dwelling

Tap in yard $\leq 10 \mathrm{~m}$ away

Tap in yard $>10$ m away

Toilet

Flush

Pit latrine $\leq 10$ m away

Pit latrine $>10$ m away $2(5.0)$ 


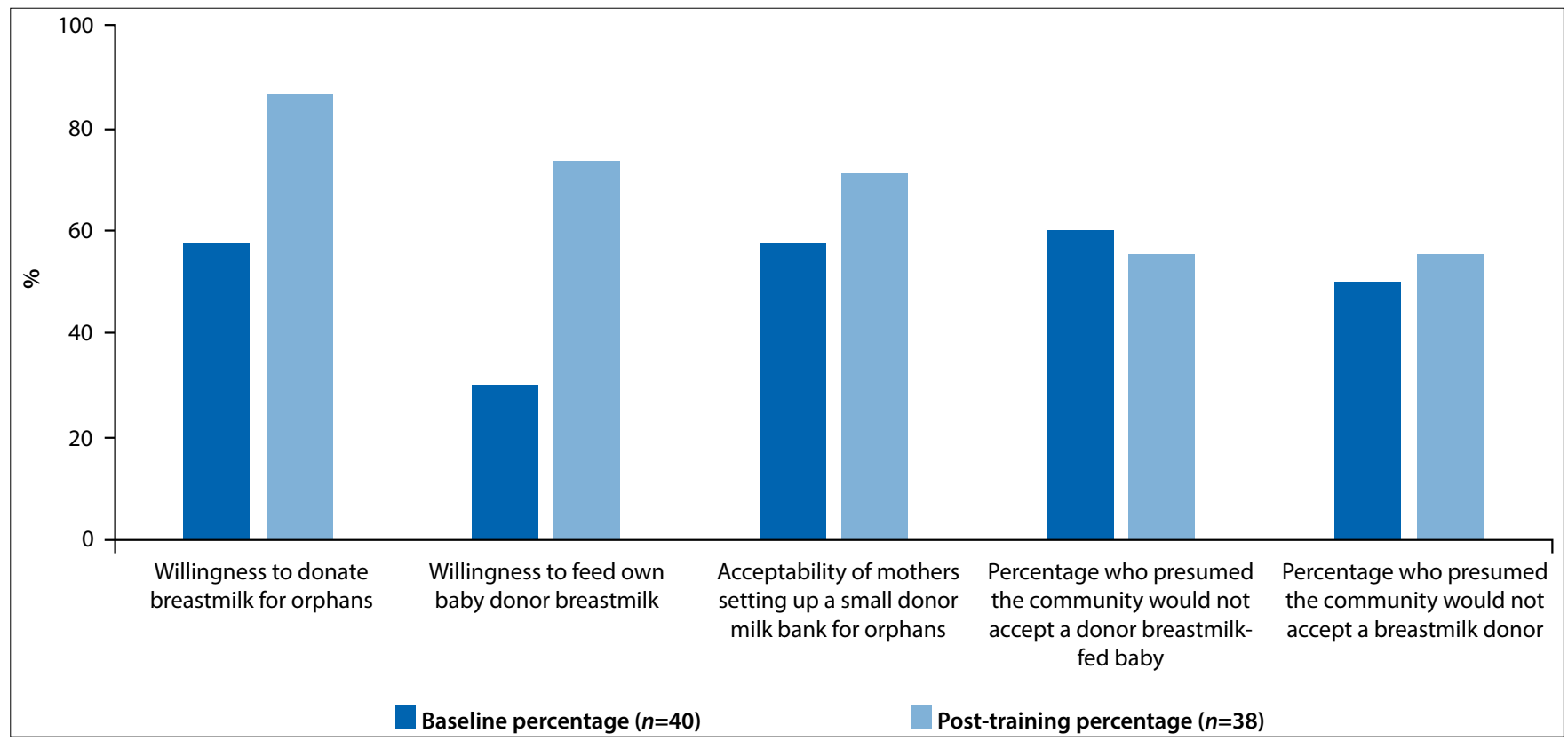

Fig. 1. Mothers' perceptions before and after training.

acceptability of HMBs within hospital settings have shown similar findings. A 2013 qualitative study in South Australia focusing on a middle-class, well-educated group of mothers found that HMBs would be well received provided the safety of donor milk was well established. ${ }^{[14]}$

The results of this study have corroborated the findings of earlier focus group discussions conducted in $\mathrm{SA},{ }^{[15]}$ which reported that obstacles to acceptability of DHM arose from lack of education about DHM and banking practices. The results of this study showed that training improved mothers' understanding of the uses and value of DHM and the processes used to ensure its safety. Before the training, some mothers showed willingness to donate milk to orphans and to set up HMBs in their own homes. Following training, this number increased, suggesting that training can complement and expand upon existing interest.

The results of this study therefore clearly demonstrate that education around breastfeeding can improve mothers' perceptions about DHM; the role of donors; and the value of HMBs; including home-based HMBs or CBHMBs. Changing perceptions of DHM among this key target population is a critical first step which could in turn impact the potential of these women to serve as donors in the future.

The results also suggest that some concerns, particularly around acceptability in the community, may persist. This may be due to mothers' fears that long-standing traditional myths associated with human milk would still be prevalent in the community. Although mothers' perceptions had changed favourably, it was clear that the mothers felt that even though they had been trained this was unlikely to have a major effect on community perceptions around milk banking. This highlights the importance, as we have seen with breastfeeding promotion, of community awareness campaigns targeting not only mothers but partners, grandparents and community leaders. ${ }^{[16]}$ In SA this would be particularly important because of the high HIV prevalence among women of reproductive age and the perceived dangers associated with breastmilk. The prevalence of HIV-infected antenatal women in KZN was $37.4 \%$ in 2012, the highest in the country and higher than the national prevalence of $29.5 \% .{ }^{[17]}$

A limitation of this study was that only women who could speak and read simple English were included. This population may not be representative of all community members, especially those in more rural, less educated settings where traditional views and myths could have an effect on perceptions of donor milk. A further limitation is the ability to generalise the results beyond this population, which was comprised of women with an interest in breastfeeding.

\section{Conclusion}

When a mother's own milk is not available, DHM provides a lifesaving second chance for many vulnerable infants. Continued research, including attention to sustainable, context-appropriate options such as CBHMBs, is key to increasing access to donor milk for those who need it. Simplified, low-cost processes designed for resourcelimited settings are being explored for pasteurisation, and additional work in this area is needed. ${ }^{[18]}$ Integrating HMB education within breastfeeding-promotion campaigns and ensuring rigorous qualitycontrol systems are crucial steps to establishing effective systems. ${ }^{[19]}$ Trust and support varies widely among cultures and communities, so targeted education and engagement remain key. The results of this study demonstrate the critical role of education training to improve awareness and acceptability of this lifesaving intervention and suggest the importance of multifaceted awareness programmes that extend beyond the mother to the wider community.

Funding and conflicts of interest. The authors declare that they have no competing interests. The authors would like to acknowledge the funders who graciously supported this work, including the Bill and Melinda Gates Foundation, through the Grand Challenge Explorations Initiative; the University of Washington Department of Computer Science and Engineering; donations from private foundations and individuals to the PATH Health Innovation Portfolio; and the National Science Foundation Research Grant No. 11S-1111433.

Acknowledgements. The authors wish to thank all the mothers who participated in the survey and Gcinile Maphanga for assisting with the interviews. We would also like to acknowledge support from the eThekwini Health Unit and the Cato Manor Clinic for allowing us to conduct the study at the latter Clinic.

\section{References}

1. Duijt L, Jaddoe VW, Hofman A, Moll HA. Prolonged and exclusive breastfeeding reduces the risk of infectious diseases in infancy. Pediatrics 2010;126(1):e18-e25. [http://dx.doi.org/10.1542/peds.2008-3256] 
2. WHO Collaborative Study Team on the Role of Breastfeeding on the Prevention of Infant Mortality. Effect of breastfeeding on infant and child mortality due to infectious diseases in less developed countries: A pooled analysis. Lancet 2000;355(9202):451-455. [http://dx.doi.org/10.1016/S0140-6736(00)82011-5]

3. Jones G, Steketee RW, Black RE, Bhutta ZA, Morris SS; and the Bellagio Child Survival Study Group. How many child deaths can we prevent this year? Lancet 2003;362(9377):65-71. [http://dx.doi.org/10.1016/S0140-6736(03)13811-1]

4. Boyd CA, Quigley MA, Brocklehurst P. Donor breast milk versus infan formula for preterm infants: Systematic review and meta-analysis. Arch Dis Child Fetal Neonatal Ed 2007;92(3):F169-F175.[http://dx.doi.org/10.1136/ adc.2005.089490]

5. Arslanoglu S, Ziegler EE, Moro GE. World Association of Perinatal Medicine (WAPM) Working Group on Nutrition. Donor human milk in preterm infant feeding: Evidence and recommendations. J Perinat Med 2010;38(4):347-351. [http://dx.doi.org/10.1515/jpm.2010.064]

6. Imdad A, Yakoob MY, Bhutta ZA. Effect of breastfeeding promotion interventions on breastfeeding rates, with special focus on developing countries. BMC Public Health 2011;11(Suppl 3):S24. [http://dx.doi.org/10.1186/14712458-11-s3-s24]

7. World Health Organization. Infant and Young Child Feeding: Model Chapter for Textbooks for Medical Students and Allied Health Professionals. Geneva WHO, 2009.

8. South African Department of Health. The Tshwane Declaration for the Support of Breastfeeding in South Africa. S Afr J Clin Nutr 2011;24(4):214

9. Statistics South Africa. Stastistical release Census 2011. http://www.statssa.gov. za/publications/P03014/P030142011.pdf (accessed 22 July 2014).

10. Coutsoudis I, Adhikari M, Nair N, Coutsoudis A. Feasibility and safety of setting up a donor breastmilk bank in a neonatal prem unit in a resource limited setting: An observational, longitudinal cohort study. BMC Public Health 2011;11(1):356. [http://dx.doi.org/10.1186/1471-2458-11-356]

11. Geraghty SR, List BA, Morrow GB. Guidelines for establishing a donor human milk depot. J Hum Lact 2010;26(1):49-52. [http://dx.doi. org/10.1177/0890334409347467]

12. Osbaldiston R, Mingle LA. Characterization of human milk donors. J Hum Lact 2007;23(4):350-357. [http://dx.doi.org/10.1177/0890334407307547]

13. Ogala WN. Attitudes of nursing mothers to breast milk banking. Niger J Paediatr 1987;14(3-4):97-101.

14. Mackenzie C, Javanparast S, Newman L. Mothers' knowledge of and attitudes toward human milk banking in South Australia: A qualitative study. J Hum Lact 2013;29(2):222-229. [http://dx.doi.org/10.1177/0890334413481106]

15. Coutsoudis I, Petrites A, Coutsoudis A. Acceptability of donated breast milk in a resource limited South African setting. Int Breastfeed J 2011;6:3. [http:// dx.doi.org/10.1186/1746-4358-6-3]

16. Shealy KR, Ruowei L, Benton-Davis S, Grummer-Strawn LM. The CDC Guide to Breastfeeding Interventions. Atlanta: US Department of Health and Human Services, Centers for Disease Control and Prevention, 2005.

17. South African National Department of Health. The 2012 National Antenatal Sentinel HIV and Herpes Simplex Type-Prevalence Survey. Pretoria: National Department of Health, 2013.

18. Chaudhri R, Vlachos D, Kaza J, et al. 2011. A system for safe flash-heat pasteurization of human breast milk. In: Proceedings of the 5th ACM workshop on Networked systems for developing regions (NSDR 2011). New York: ACM, 2011:9-14. [http://dx.doi.org/10.1145/1999927.1999932]

19. PATH. Strengthening Human Milk Banking: A Global Implementation Framework. Version 1. Seattle: Bill and Melinda Gates Foundation Grand Challenges initiative, PATH, 2013. 